\title{
Downregulation of coding transmembrane protein 35 gene inhibits cell proliferation, migration and cell cycle arrest in osteosarcoma cells
}

\author{
YINJUN HUANG* , SHICHANG ZHAO* , YADONG ZHANG, CHANGQING ZHANG and XIAOLIN LI \\ Department of Orthopedic Surgery, Shanghai Sixth People's Hospital, \\ Shanghai Jiao Tong University, Shanghai 200233, P.R. China
}

Received December 9, 2014; Accepted February 26, 2016

DOI: $10.3892 /$ etm.2016.3381

\begin{abstract}
Osteosarcoma (OSA) is the most common primary tumor of the bone. Resistance to chemotherapy and the fast rapid development of metastatic lesions are major issues responsible for treatment failure and poor survival rates in OSA patients. Tetraspanins comprise a family of transmembrane receptor glycoproteins that affect tumor cell migration through tetraspanin-integrin interaction. The present study focused on a four-pass transmembrane protein gene, transmembrane protein 35 (TMEM35) gene, and examined its role in the growth, migration and cell cycle progression of OSA cells. In addition, the study discussed whether the TMEM35 gene, which encodes the TMEM35 protein, may be a potential therapeutic target for OSA. In the current study, reverse transcription-quantitative polymerase chain reaction was performed to examine TMEM35 expression in OSA and matched healthy tissues. Small interfering RNAs (siRNAs) were transfected into $\mathrm{SaOS} 2$ and U2OS cells to knockdown the TMEM35 expression. Soft-agar colony formation assay was performed to evaluate cell growth, and cell cycle progression was analyzed by flow cytometry. Wound-healing and Boyden chamber assays were also performed to investigate cell invasion and migration by the SaOS2 and U2OS cells. TMEM35 protein was analyzed in a functional protein interaction
\end{abstract}

Correspondence to: Professor Xiaolin Li, Department of Orthopedic Surgery, Shanghai Sixth People's Hospital, Shanghai Jiao Tong University, 600 Yishan Road, Shanghai 200233, P.R. China E-mail: lixiaolin@sjtu.edu.cn

Abbreviations: OSA, osteosarcoma; TMEM35, transmembrane protein 35; RT-qPCR, reverse transcription-quantitative polymerase chain reaction; NPY, neuropeptide Y; NGF, nerve growth factor; NGFR, nerve growth factor receptor; BDNF, brain-derived neurotropic factor; CALCB, calcitonin related polypeptide $\beta$; VIP, vasoactive intestinal peptide

${ }^{*}$ Contributed equally

Key words: RNA interference, tetraspanin, osteosarcoma, cell cycle networks database (STRING database) to predict the functional interaction partner proteins of TMEM35. The results indicated that TMEM35 was abnormally expressed in OSA tissues. Of the 37 examined patients, TMEM35 expression was significantly increased in the OSA tissues of 24 patients $(64.86 \%$; $\mathrm{P}<0.05)$, when compared with the expression in normal tissues. Furthermore, TMEM35 knockdown following transfection with siRNAs inhibited the colony formation ability of SaOS2 and U2OS cells in soft agar. Flow cytometric analysis also revealed that TMEM35 knockdown by RNA interference may result in G1 phase arrest and a decreased cell population at the S phase. TMEM35 knockdown inhibited cell migration in SaOS2 and U2OS cells in wound-healing assays. In conclusion, TMEM35, a member of the tetraspanin family, serves an important role in the growth of OSA cells.

\section{Introduction}

Osteosarcoma (OSA) is a common type of primary tumor of the bone (1-3). OSA has similar worldwide incidence rates in children and adolescents; the incidence rate is 5.5-14 per million persons at age 15-19 years (4). The 5-year survival rate in patients presenting with localized OSA is $~ 80 \%$, whereas the prognosis is poor in patients with metastatic disease (5). Primary tumors commonly originate from the proximal tibia and humerus, as well as from the metaphyseal (actively growing) regions of the distal femur; however, OSA may develop in any bone of the body, with lungs and bone being the most likely sites of metastasis (5).

Tetraspanins are a set of transmembrane receptor glycoproteins with a molecular weight between 25 and $50 \mathrm{kDa}$. They are involved in numerous important physiological processes (6-14). Tetraspanins have been found to be associated with the expression of various tumor prognosis factors, such as CD9 in lung cancer (15-17), CD82 in prostatic cancer (18-20), and CD63 and CD9 in melanoma $(21,22)$. Decreased tetraspanin expression contributes to the promotion of tumor invasion through the lymphatic system. It had been reported that downstream regulation of tetraspanin is critical for tumor progression in breast cancer (23).

Coding transmembrane protein 35 (TMEM35) is a transmembrane protein that is conservatively expressed in 
humans, canines, cattle, mice, rats, chicken, zebra fish and Drosophila species (24); however, the function of TMEM35 remains poorly understood. A previous study in rats revealed that TMEM35 may be a candidate regulatory factor involved in adrenal cortex-zona glomerulosa growth following sodium consumption (24).

In the present study, the TMEM35 expression in OSA tissues and cell lines were investigated, as well as the effect of TMEM35 knockdown on cell cycle progression.

\section{Materials and methods}

Cell culture. Two OSA cell lines, SaOS2 and U2OS (American Type Culture Collection, Manassas, VA, USA) were investigated in the present study. Cells were cultured in Dulbecco's modified Eagle medium (DMEM; Invitrogen; Thermo Fisher Scientific, Inc., Paisley, Scotland) containing $10 \%$ fetal calf serum, $10,000 \mathrm{U} / \mathrm{ml}$ penicillin and $10,000 \mathrm{mg} / \mathrm{ml}$ streptomycin, at $37^{\circ} \mathrm{C}$ in a humidified atmosphere containing $5 \% \mathrm{CO}_{2}$ in air.

Human tissues. Tissue samples were collected from 37 patients diagnosed with OSA at the Department of Orthopedic Surgery, Shanghai Sixth People's Hospital (Shanghai, China). All specimens were acquired from patients who underwent surgical resection and provided informed consent. Among the 37 patients, 25 were male and 12 were female. The ages of the patients ranged from 14-45 years. Specimens of tumor and adjacent normal tissue were collected from each patient, and the diagnosis of OSA was validated by pathological examination. Specimens were frozen at $-80^{\circ} \mathrm{C}$ for DNA/RNA extraction. The Ethics Committee of Shanghai Sixth People's Hospital provided ethical approval.

RNA extraction and reverse transcription-quantitative polymerase chain reaction ( $R T-q P C R)$. Total RNA was isolated from cells or tissues using TRIzol reagent (Invitrogen; Thermo Fisher Scientific, Inc., Cergy Pontoise, France). Next, $3 \mathrm{mg}$ total RNA was denatured for $10 \mathrm{~min}$ at $70^{\circ} \mathrm{C}$ and then reversed transcribed into cDNA at $37^{\circ} \mathrm{C}$ for $90 \mathrm{~min}$ using $300 \mathrm{U}$ Moloney murine leukemia virus reverse transcriptase, $15 \mathrm{mg}$ oligo dT primers (both Invitrogen; Thermo Fisher Scientific, Inc., Waltham, MA, USA) and $1 \mathrm{mM}$ deoxynucleoside triphosphate (Bioline, London, UK) in a total volume of $30 \mathrm{ml}$. qPCR was then performed using a SYBR Green PCR Master Mix kit (ABgene; Thermo Fisher Scientific, Inc., Courtaboeuf Cedex, France) supplemented with $0.5 \mathrm{mM}$ primers. The PCR mixture contained 7.5 $\mu \mathrm{l}$ SYBR Green, $4.5 \mu \mathrm{l}$ water, $1 \mu \mathrm{l}$ forward and reverse primers, respectively, and $2 \mu \mathrm{l}$ DNA template. The primers were: Human TMEM35 forward, 5'-TGGGGACTATCAAGCTGACC-3', and reverse, 5'-CAATGCTTTTTCGGAGGAGA-3'; $\beta$-actin forward, 5'-AATCGTGCGTGACATTAAGGAG-3', and reverse, 5'-ACTGTGTTGGCGTACAGGTCTT-3'. The thermal cycling conditions used were as follows: $95^{\circ} \mathrm{C}$ for $15 \mathrm{~min}$, then 40 cycles at $95^{\circ} \mathrm{C}$ for $20 \mathrm{sec}, 58^{\circ} \mathrm{C}$ for $15 \mathrm{sec}$, and $72^{\circ} \mathrm{C}$ for $15 \mathrm{sec}$. Signals with a threshold cycle $(\mathrm{Cq})$ value of $>39$ were considered to indicate no transcription of the target gene. The relative expression of mRNA was calculated using the $2^{-\Delta \Delta \mathrm{Cq}}$ method (25).
Cell transfection. siRNAs specifically targeting the TMEM35 gene (TMEM35-si-1 and TMEM35-si-2), and negative control (si-LUC) were transfected into SaOS2 and U2OS cells. The siRNA sequences were TMEM35-si-1: CCAGAACCG UAACUAUUGU and TMEM35-si-2: CAACCCUCCUUA UAUGAGA. The siRNAs and Lipofectamine 2000 (both Invitrogen) were combined in DMEM at room temperature. The mixture was added to the cells dropwise and incubated for 4-6 h. The medium was then changed to fresh medium containing $10 \%$ fetal bovine serum (FBS; Gibco; Thermo Fisher Scientific, Inc., Waltham, MA, USA) and 1\% antibiotic, and cells were cultured for 3 days.

Wound-healing and cell migration assays. A wound-healing assay was performed using $\mathrm{SaOS} 2$ cells according to the manufacturer's protocol (ibidi GmbH, Martinsried, Germany). Cell migration assays were also performed using SaOS2 and U2OS cell lines, as described previously (26). Briefly, in the wound-healing assay, cells were seeded into 3-mm cell culture dishes and when cell confluence was $\sim 90 \%$, a scratch was made at the bottom of the dish. The width of the wound was measured to assess wound healing. The cell migration assay was a Boyden chamber assay in which cells were seeded in an insert with a porous membrane (50,000 cells/insert). DMEM without FBS was added to the upper chamber and DMEM with $10 \%$ FBS was added to the bottom of the plate. The insert was put into the well of a 12 well plate containing medium for $24 \mathrm{~h}$. The number of cells that transferred to the bottom of the membrane from the upper side was determined. To do this, non-migrated cells were removed from the upper surface using cotton-tipped swabs. Cells that migrated to the underside were fixed with $3.7 \%$ paraformaldehyde in phosphate-buffered saline at $4^{\circ} \mathrm{C}$ and stained with crystal violet. Membranes were then cut from the insert and observed under a microscope. Five fields were randomly selected and a count for each assay was performed in duplicate.

Colony formation assays. A total of $\sim 20,000 \mathrm{SaOS} 2$ or $\mathrm{U} 2 \mathrm{OS}$ cells were seeded into dishes and cultured at $37^{\circ} \mathrm{C}$ for 2 weeks in DMEM containing $10 \%$ fetal bovine serum and $1 \%$ antibiotic. Soft agar colony formation assays were conducted, in which the transfected cells were seeded in soft agar and incubated for approximately 3-4 weeks. At the end of the incubation, the cells were fixed with $4 \%$ paraformaldehyde and stained with $0.1 \%$ crystal violet (Hangzhou DayangChem Co., Ltd.; Hangzhou, China) or 1\% Coomassie Brilliant Blue. The colonies were then counted under a dissecting microscope (AmScope SE306-AZ-E; AmScope, Irvine, CA, USA).

Cell cycle analyses. Flow cytometry with propidium iodide staining was used to analyze cell cycle progression. The cells were fixed in $70 \%$ ethanol and rehydrated in phosphate-buffered saline and adjusted to a concentration of $1-5 \times 10^{6}$ cells $/ \mathrm{ml}$. Cells were treated with $10 \mathrm{mg} / \mathrm{ml}$ RNase A for $30 \mathrm{~min}$ and $10 \mu \mathrm{g} / \mathrm{ml}$ PI (both Sigma-Aldrich, St. Louis, MO, USA) for $15 \mathrm{~min}$ at room temperature. The cells were kept in the dark on ice or at $4^{\circ} \mathrm{C}$ in a fridge until analysis. Results were analyzed using a BD Accuri C6 Plus flow cytometer with Flowjo software (BD Biosciences, San Jose, CA, USA). 


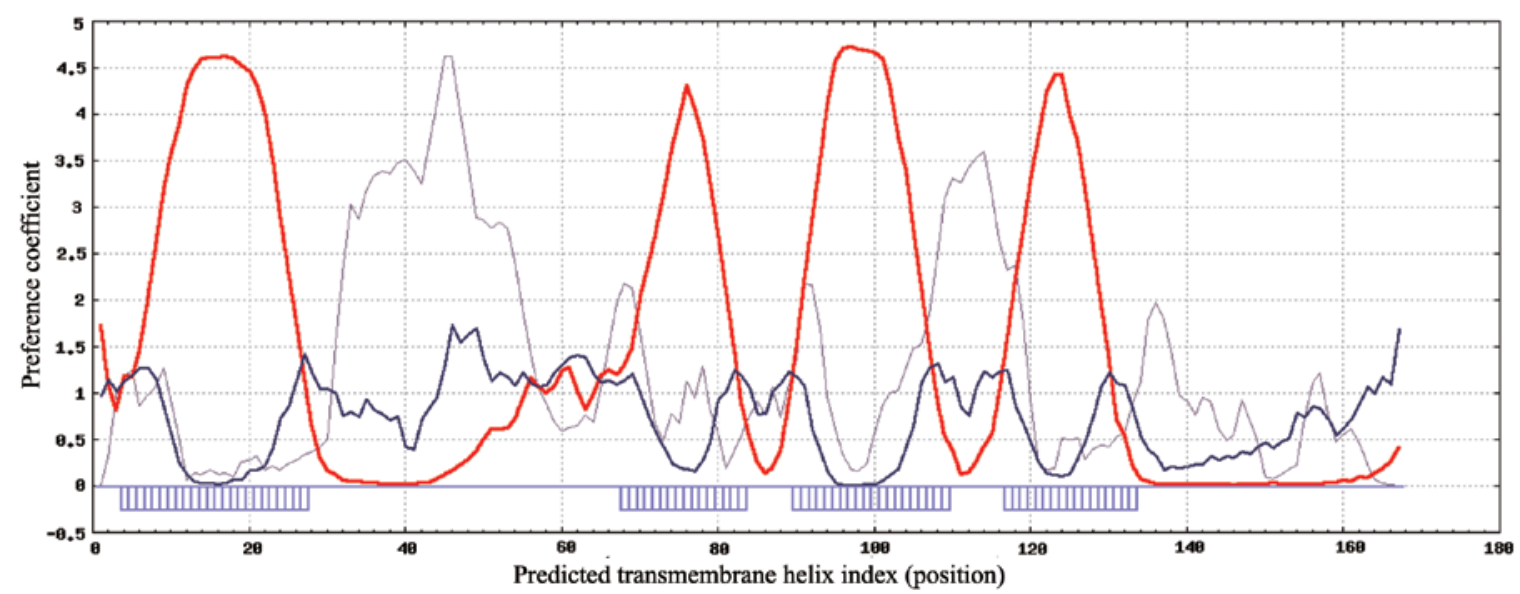

Figure 1. Secondary structure of TMEM35 analyzed using SPLIT software, version 4.0. Red line, transmembrane helix preference coefficient; blue line, $\beta$ preference coefficient; gray line, modified hydrophobic moment index; violet boxes, predicted transmembrane helix position index.

Bioinformatics analysis. In order to analyze the structural characteristics and secondary structure of TMEM35, SPLIT software version 4.0 (split.pmfst.hr/split/4/) was used (27). For investigation of the poorly understood TMEM35 function and the associated molecular mechanism, the STRING database (http://string-db.org) (27) was used to predict the proteins interacting with TMEM35. The database contains known and predicted protein interactions, including directly (physical) and indirectly (functional) relevant interactions. The data are derived from four sources: Genomic context, high-throughput experiments, coexpression and previous knowledge.

Statistical analysis. Two-factor analysis of variance was performed to compare the data. Student's t-test was used to determine the statistical significance. The results are presented as mean \pm standard deviation. SPSS statistical analysis software, version 21.0 (IBM SPSS, Armonk, NY, USA) was used to analyze the results. $\mathrm{P}<0.05$ was considered to indicate a statistically significant difference.

\section{Results}

Human TMEM35 gene. SPLIT software was used to analyze the structural characteristics and secondary structure of TMEM35 (27). On the basis of the results presented in Fig. 1, TMEM35 is a low molecular weight, cell membrane surface protein with four highly hydrophobic domains. TMEM35 has a structure typical of a tetraspanin, the functions of which have been reviewed previously (28), and include extensive involvement in cell proliferation, adhesion and migration. Therefore, we hypothesize that cell signal transduction pathways are directly or indirectly influenced by TMEM35, due to an effect on tumor cell adhesion, differentiation, migration and invasion.

Upregulation of TEME35 in OSA cells. In order to investigate the TMEM35 expression in OSA, RT-qPCR assays were performed in human OSA samples and non-tumor tissues. As shown in Fig. 2, TMEM35 was found to be upregulated in $24 / 37$ (64.86\%) OSA samples, among which 22 samples presented a 2-fold upregulation of TMEM35 (two tailed t-test,
$\mathrm{P}<0.05)$. These results indicated that TMEM35 overexpression may play an important role in OSA.

Knockdown of TMEM35 expression inhibits OSA cell growth. To further investigate the effects of TMEM35 expression on OSA cell growth, TMEM35 knockdown was performed by transfection with siRNA, and the effect of TMEM35 knockdown on soft agar colony formation was evaluated. siRNAs specifically targeting the TMEM35 gene (TMEM35-si-1 and TMEM35-si-2) were transfected into SaOS2 and U2OS cells, and RT-qPCR was performed to evaluate the efficiency of gene knockdown. The results indicated that TMEM35 expression decreased significantly following transfection, indicating that the siRNAs silenced TMEM35 expression in the SaOS2 and U2OS cells (Fig. 3A). Subsequently, the effect of TMEM35 knockdown on OSA cell colony formation was examined.

A soft agar colony formation assay of SaOS2 and U2OS cells was performed to evaluate the contact inhibition effect on these tumor cells, and the results are shown in Fig. 3B. Results indicated that the number and size of the cell colonies decreased significantly compared with those in the negative control (si-LUC). Subsequently, the number of SaOS2 and U2OS cell colonies were investigated with crystal violet staining (Fig. 3C). Results demonstrated that TMEM35 knockdown significantly inhibited SaOS2 and U2OS cell growth in the soft agar (two tailed t-test, $\mathrm{P}<0.05$ ), implying that TMEM35 may play an role in the adherence-independent growth of OSA cells.

TMEM35 knockdown arrests OSA cells in G1 phase. In order to explain how the cytological metabolism of TMEM35 knockdown inhibits cell growth in OSA cells, flow cytometric analysis was performed to analyze changes in the cell cycle progression following TMEM35 silencing in SaOS2 cells. Cells transfected with either si-LUC or TMEM35-si-1 were collected and stained with PI. As shown in Fig. 4, the proportion of cells in the G0/G1 phase increased (si-LUC, 61.07 $\pm 3.21 \%$; TMEM35-si-1, 73.35 $\pm 4.52 \%$ ), and the proportion of cells in the $\mathrm{S}$ phase decreased (si-LUC, 28.16 $\pm 1.23 \%$; TMEM35-si-1, $14.55 \pm 2.31 \%$ ) when compared with the cell population in the negative control (si-LUC) following TMEM35 silencing in 
A

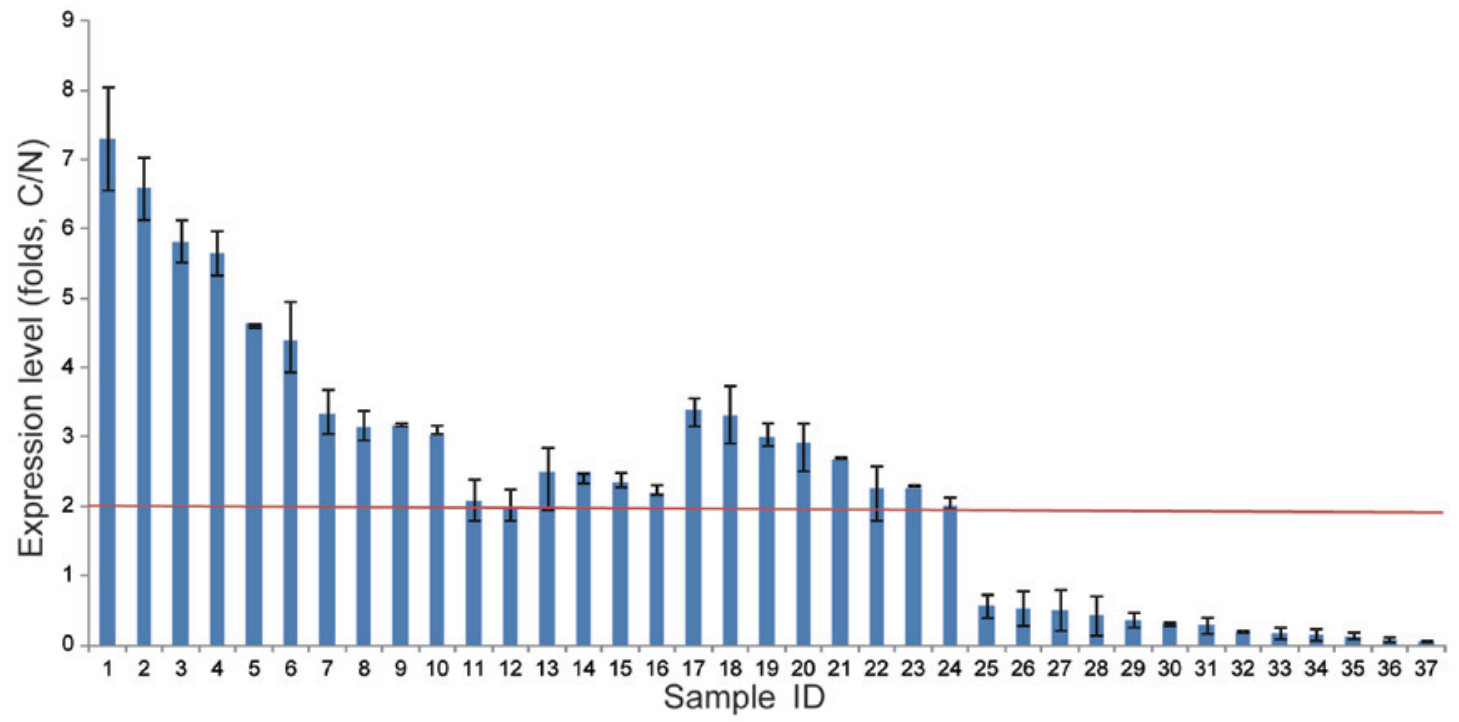

B
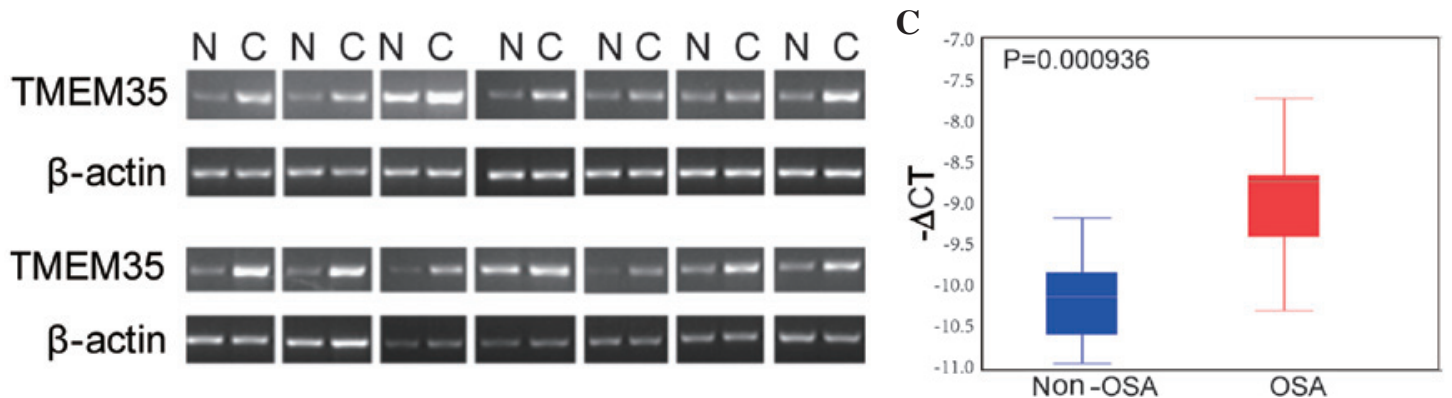

Figure 2. TMEM35 expression in OSA samples. (A) Expression level changes in OSA samples compared with non-tumor tissues evaluated by quantitative polymerase chain reaction. (B) Examples of expression level differences in OSA samples compared with non-tumor tissues evaluated by reverse transcription polymerase chain reaction. (C) Statistical analysis of TMEM35 expression in OSA. OSA, osteosarcoma; TMEM35, transmembrane protein 35; C, tumor tissue; $\mathrm{N}$, non-tumor tissue.

SaOS 2 cells. These collective data indicate that TMEM35 knockdown reduced the $\mathrm{S}$ phase cell population and resulted in $\mathrm{G} 1$ arrest in SaOS2 cells, which suggests that TMEM35 participated in cell cycle progression.

In vitro cell migration depends on TMEM35 expression. The migratory potential of transduced OSA cell lines was determined, since metastasis is the main deleterious characteristic of OSA cells. The results indicated that cell migration was reduced subsequent to TMEM35 silencing when compared with that in the parental cells (Fig. 5A). A Boyden chamber assay confirmed the strong association between TMEM35 expression and migratory potential (Fig. 5B and C). These data indicated that TMEM35 expression regulates the migratory potential, in vitro cell migration and invasive abilities of human OSA cells. In addition, the results demonstrated that a reduced TMEM35 expression attenuates the OSA cell aggressiveness.

Identification of potential functional partners of TMEM35 protein. For investigation of the poorly understood TMEM35 function and the associated molecular mechanism, the STRING database (27) was used to predict the proteins interacting with TMEM35. The database contains known and predicted protein interactions, including directly (physical) and indirectly (functional) relevant interactions. As demonstrated in Fig. 6, TMEM35 may be associated with neuropeptide Y
(NPY), nerve growth factor (NGF), NGF receptor (NGFR), brain-derived neurotropic factor (BDNF), vasoactive intestinal peptide (VIP) and calcitonin-related polypeptide $\beta$ (CALCB). These predicted functional partner proteins indicate that TMEM35 may play an important role in cell differentiation and survival, further revealing the important function of TMEM35.

\section{Discussion}

Transmembrane proteins, coded by $\sim 30 \%$ of genes in humans, play critical roles in human physiological and pathological progression (29). Transmembrane proteins have been reported to be directly associated with numerous serious human diseases, including Alzheimer's disease and cardiovascular diseases (30). Approximately $50 \%$ of drugs for such diseases target transmembrane proteins (31), which highlights the importance of further investigation into the structure and function of transmembrane proteins. TMEM35 is a four-pass transmembrane protein that exists conservatively in humans, canines, cattle, mice, rats, chicken, zebra fish and Drosophila species (24). A previous study in rats revealed that TMEM35 may be a candidate regulatory factor involved in adrenal cortex-zona glomerulosa growth following sodium consumption (24). However, the function of TMEM35 in OSA remains poorly understood. It has been reported that another four-pass 
A

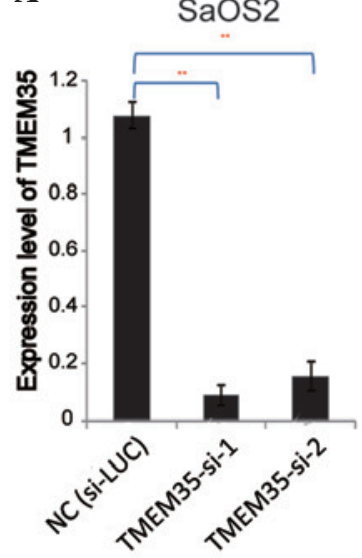

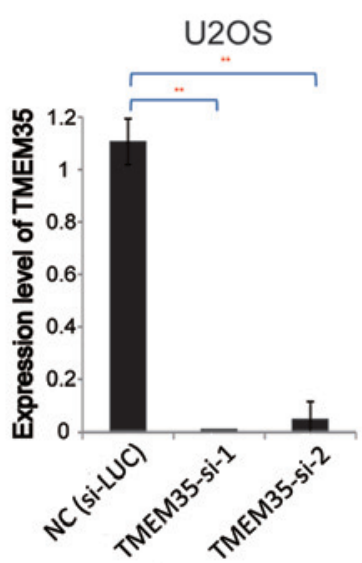

C

$\mathrm{SaOS} 2$
B
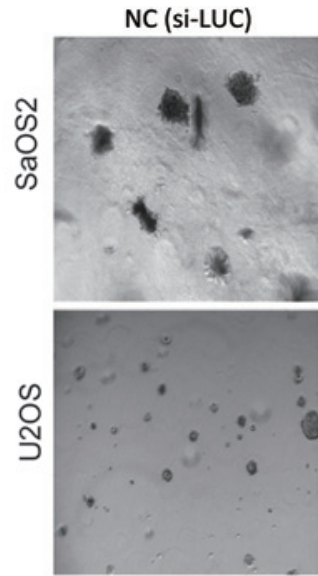

TMEM35-si-1
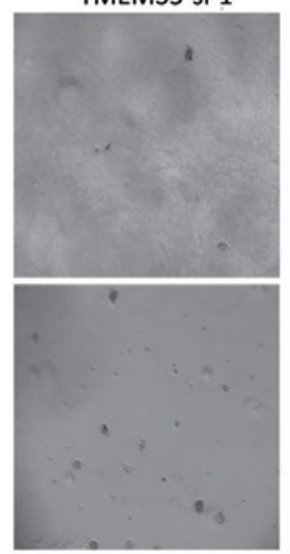

U2OS
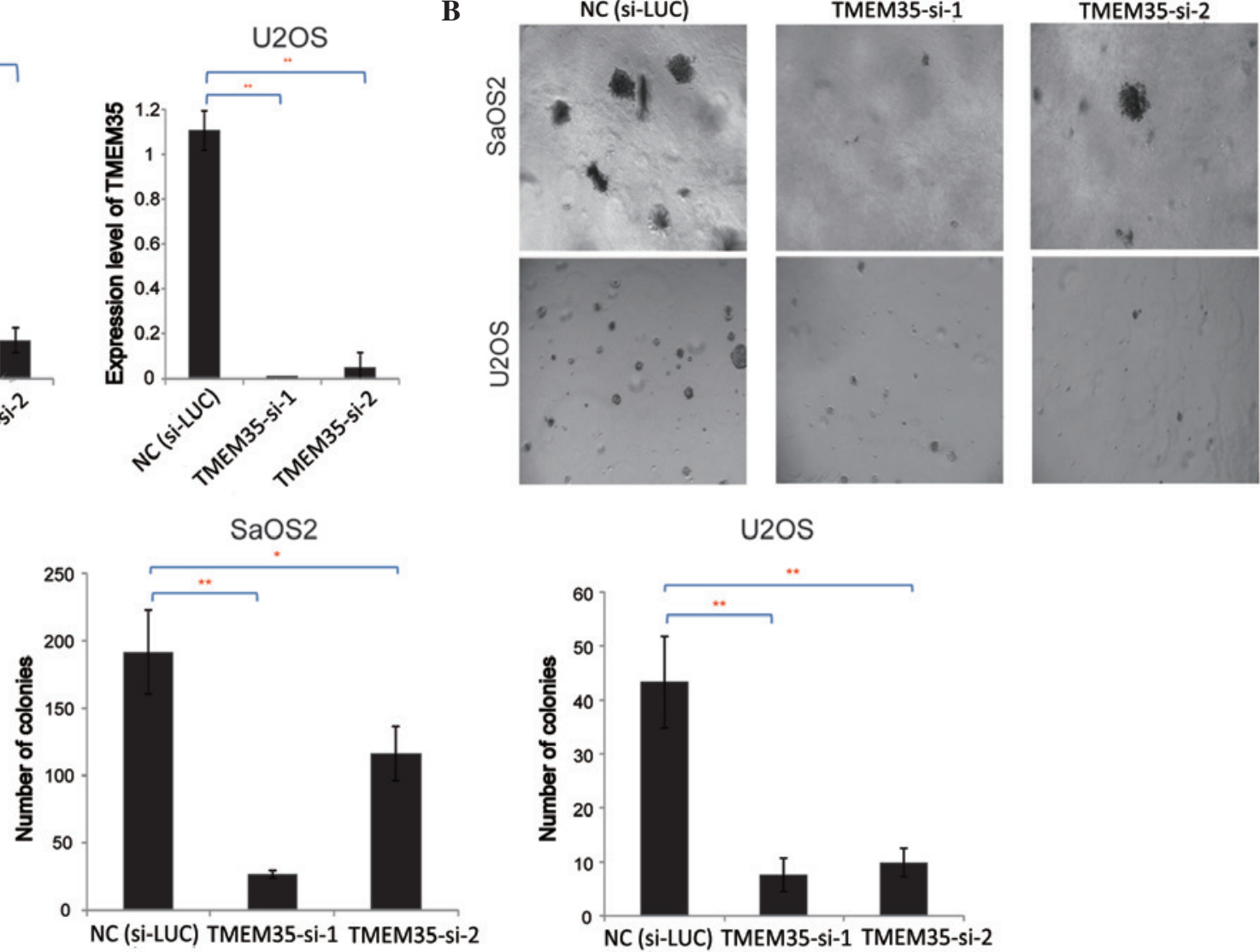

Figure 3. TMEM35 knockdown inhibits soft agar colony formation in SaOS2 and U2OS cells. (A) Silencing efficiency of TMEM35-si-1 and -si-2 compared with negative control (NC; si-LUC) in SaOS2 and U2OS cells evaluated by reverse transcription-quantitative polymerase chain reaction. (B) Soft agar colony formation after TMEM35 knockdown in the two cell lines. (C) Statistical analysis of number of clones in each group. ${ }^{*} \mathrm{P}<0.05,{ }^{* *} \mathrm{P}<0.01$. TMEM 35 , transmembrane protein 35 .

A

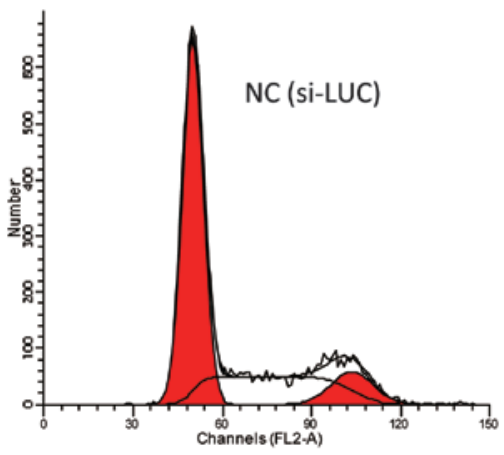

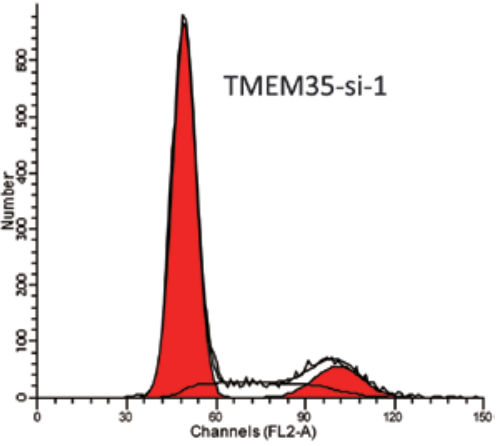

B

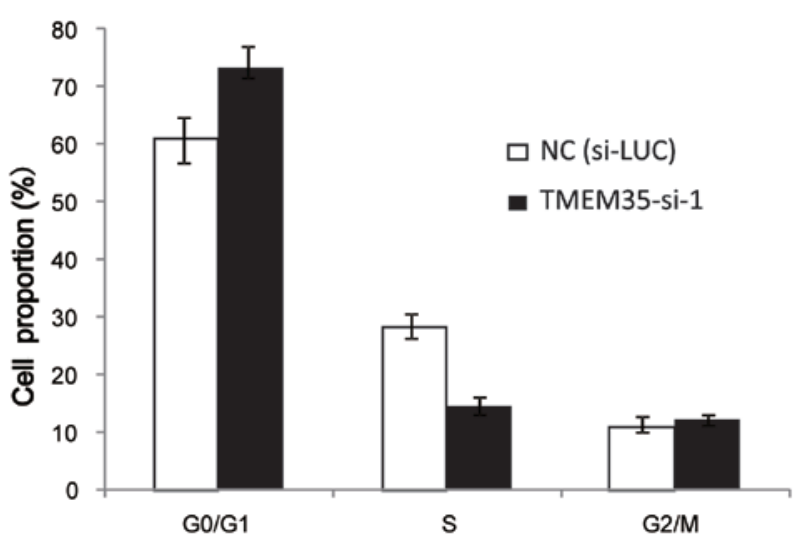

Figure 4. TMEM35 knockdown arrests osteosarcoma cells in the G1 phase. (A) Flow cytometry was performed to analyze changes in the cell cycle after TMEM35 was silenced with TMEM35-si-1 in SaOS2 cells, and compared with. negative control (NC; si-LUC). (B) Statistical analysis of cell proportion in different cell cycle phases. TMEM35, transmembrane protein 35. 
A

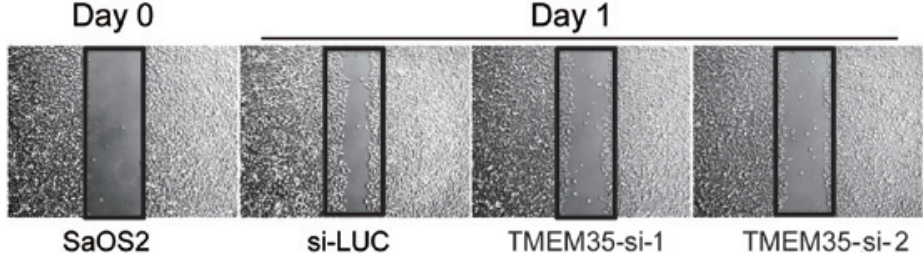

B

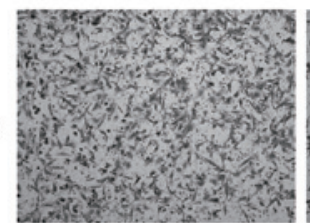

Cell Migration

SaOS2

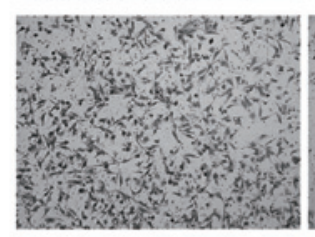

si-LUC
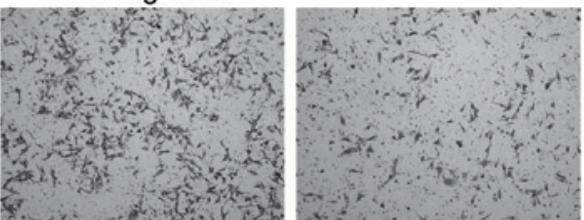

U2OS

C
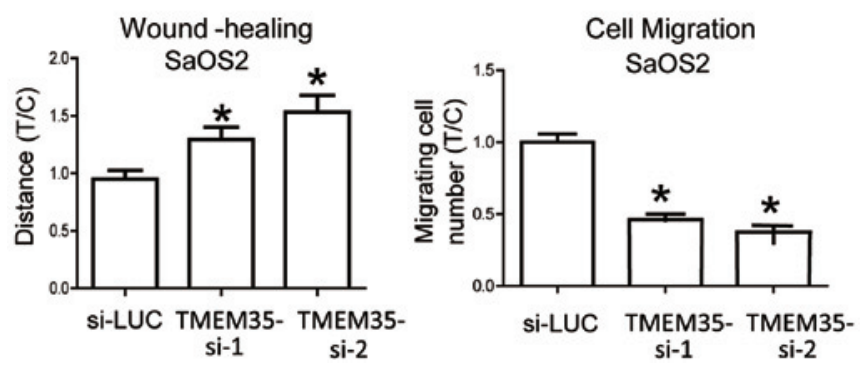

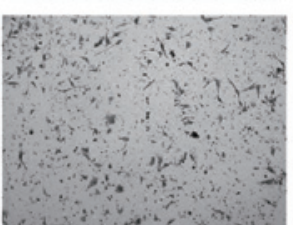

TMEM35-si-1

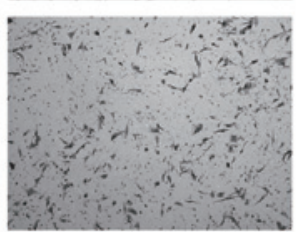

TMEM35-si-2

Figure 5. Osteosarcoma cell migration was controlled by TMEM35 expression. (A) Quantification of SaOS2 cell migration on day 1 after wounding. Representative phase-contrast images on days 0 and 1 after wounding. (B) Migrated cells were stained and counted after SaOS2 and U2OS cells were seeded in chamber for migrating for $24 \mathrm{~h}$. (C) Analysis of migrated cells after wounding and migration. " $\mathrm{P}<0.05$ vs. parental cells. TMEM 35 , transmembrane protein 35 ; $\mathrm{T} / \mathrm{C}$, treatment group to control group ratio.

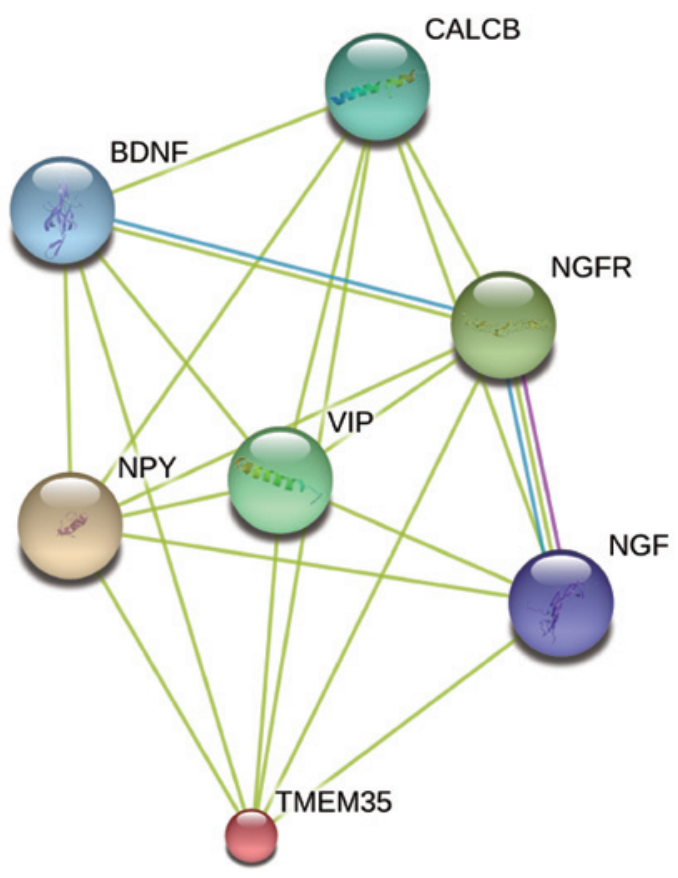

Figure 6. Prediction of potential functional partner factors of TMEM35 by the STRING database. TMEM35, transmembrane protein 35; NPY, neuropeptide Y; NGF, nerve growth factor; NGFR, NGF receptor; BDNF, brain-derived neurotropic factor; VIP, vasoactive intestinal peptide; CALCB, calcitonin-related polypeptide $\beta$. transmembrane protein gene, CD151 is highly associated with hepatocellular carcinoma invasion and metastasis (32), and is significantly upregulated in various tumors, including breast, prostate, colorectal and pancreatic cancer, as well as in hepatocellular carcinoma $(33,34)$, compared with corresponding normal tissues. Furthermore, survival and relapse rates are significantly decreased or increased, respectively, upon CD151 upregulation.

The human TMEM35 gene comprises 167 amino acids and is located at the chromosome position Xq22.1. The molecular weight and isoelectric point of TMEM35 are 18,440.23 Da and 10.09, respectively (http://www.genecards.org/). The present study investigated the expression of the transmembrane protein TMEM35, and the results indicated that it was upregulated in OSA samples compared with the normal tissues, as evaluated with RT-qPCR. TMEM35 was found to be important for OSA cell growth, and its knockdown inhibited cell growth and adherence-independent growth in soft agar. As shown in Fig. 4, TMEM35 knockdown inhibited cell cycle progression and resulted in cell cycle arrest at G1 phase, which may account for the inhibition in OSA cell growth. Migration was also inhibited by TMEM35 knockdown in OSA cells. The aforementioned results imply that TMEM35 plays an important role in OSA cell growth, migration and invasion through regulation of the cell cycle progression, 
which indicates that it may be a potential novel therapeutic target for drug development.

Furthermore, the present study investigated the potential functional partners of TMEM35. The results indicated that TMEM35 may be associated with NPY, NGF, NGFR, BDNF, VIP and CALCB. NPY is involved in the regulation of the gonadotropin-releasing hormone transport and release (35). In addition, NGF plays an important role in the development and maintenance of the sympathetic nerve and sensorium nerve system. Its receptor, NGFR, is a member of the tumor necrosis factor receptor superfamily, which binds to NGF with a low affinity (36). BDNF promotes the survival and differentiation of neurons in the peripheral and central nervous systems during the development process (37). Furthermore, VIP induces vasodilatation and reduces arterial blood pressure (38), and CALCB is also known to induce vasodilatation (39). These observations may improve the understanding of the molecular signaling pathway and mechanisms involved in the development of OSA.

In conclusion, the present study found that TMEM35 kncockdown inhibited OSA cell proliferation by arresting the cell cycle at the G1 phase. Furthermore, TMEM35 knockdown inhibited OSA cell migration. These results provide new insight into the function of TMEM35 in OSA initiation and progression. However, the precise mechanism by which TMEM35 regulates cell proliferation and migration requires further clarification. Further investigation of the role TMEM35 in the cell cycle is worthy of investigation in future studies.

\section{References}

1. Hashimoto K, Hatori M, Hosaka M, Watanabe M, Hasegawa T and Kokubun S. Osteosarcoma arising from giant cell tumor of bone ten years after primary surgery: A case report and review of the literature. Tohoku J Exp Med 208: 157-162, 2006.

2. Hayashi K, Zhao M, Yamauchi K, Yamamoto N, Tsuchiya H, Tomita K, Kishimoto H, Bouvet M and Hoffman RM. Systemic targeting of primary bone tumor and lung metastasis of high-grade osteosarcoma in nude mice with a tumor-selective strain of Salmonella typhimurium. Cell Cycle 8: 870-875, 2009

3. Takeuchi A, Yamamoto N, Shirai T, Nishida H, Hayashi K, Watanabe K, Miwa S, and Tsuchiya H. Successful correction of tibial bone deformity through multiple surgical procedures, liquid nitrogen-pretreated bone tumor autograft, three-dimensional external fixation, and internal fixation in a patient with primary osteosarcoma: A case report. BMC Surg 15: 124, 2015.

4. Stiller CA. International patterns of cancer incidence in adolescents. 33: 631-645, 2007.

5. Zhang Y, Zhang L, Zhang G, Li S, Duan J, Cheng J, Ding G, Zhou C, Zhang J, Luo P, et al: Osteosarcoma metastasis: Prospective role of ezrin. Tumour Biol 35: 5055-5059, 2014.

6. García-López MA, Barreiro O, García-Diez A, Sánchez-MadridF and Peñas PF. Role of tetraspanins CD9 and CD151 in primary melanocyte motility. J Invest Dermatol 125: 1001-1009, 2005.

7. Gartlan KH, Belz GT, Tarrant JM, Minigo G, Katsara M, Sheng KC, Sofi M, van Spriel AB, Apostolopoulos V, Plebanski M, et al. A complementary role for the tetraspanins CD37 and Tssc6 in cellular immunity. J Immunol 185 : 3158-3166, 2010

8. Goschnick MW and Jackson DE. Tetraspanins - structural and signalling scaffolds that regulate platelet function. Mini Rev Med Chem 7: 1248-1254, 2007.

9. Gourgues M, Clergeot PH, Veneault C, Cots J, Sibuet S, Brunet-Simon A, Levis C, Langin T and Lebrun MH. A new class of tetraspanins in fungi. Biochem Biophys Res Commun 297: 1197-1204, 2002.

10. Jiang $\mathrm{X}$, Zhang $\mathrm{J}$ and Huang $\mathrm{Y}$. Tetraspanins in cell migration. Cell Adh Migr 9: 406-415, 2015.

11. Jones EL, Demaria MC and Wright MD. Tetraspanins in cellular immunity. Biochem Soc Trans 39: 506-511, 2011.
12. Köberle M, Kaesler S, Kempf W, Wölbing F and Biedermann T. Tetraspanins in mast cells. Front Immunol 3: 106, 2012

13. Krementsov DN, Weng J, Lambelé M, Roy NH and Thali M. Tetraspanins regulate cell-to-cell transmission of HIV-1. Retrovirology 6: 64, 2009.

14. Perron JC and Bixby JL. Tetraspanins expressed in the embryonic chick nervous system. FEBS Lett 461: 86-90, 1999.

15. Higashiyama M, Taki T, Ieki Y, Adachi M, Huang CL, Koh T, Kodama K, Doi O and Miyake M: Reduced motility related protein-1 (MRP-1/CD9) gene expression as a factor of poor prognosis in non-small cell lung cancer. Cancer Res 55: 6040-6044, 1995.

16. Zheng R, Yano S, Zhang H, Nakataki E, Tachibana I, Kawase I, Hayashi S and Sone S: CD9 overexpression suppressed the liver metastasis and malignant ascites via inhibition of proliferation and motility of small-cell lung cancer cells in NK cell-depleted SCID mice. Oncol Res 15: 365-372, 2005.

17. Saito Y, Tachibana I, Takeda Y, Yamane H, He P, Suzuki M, Minami S, Kijima T, Yoshida M, Kumagai T, et al: Absence of CD9 enhances adhesion-dependent morphologic differentiation, survival and matrix metalloproteinase- 2 production in small cell lung cancer cells. Cancer Res 66: 9557-9565, 2006.

18. Park JJ, Jin YB, Lee YJ, Lee JS, Lee YS, Ko YG and Lee M KAI1 suppresses HIF-1 $\alpha$ and VEGF expression by blocking CDCP1-enhanced Src activation in prostate cancer. BMC Cancer 12: 81, 2012.

19. Jee B, Jin K, Hahn JH, Song HG and Lee H: Metastasis-suppressor KAI1/CD82 induces homotypic aggregation of human prostate cancer cells through Src-dependent pathway. Exp Mol Med 35: 30-37, 2003.

20. Lee HA, Park I, Byun HJ, Jeoung D, Kim YM and Lee H: Metastasis suppressor KAI1/CD82 attenuates the matrix adhesion of human prostate cancer cells by suppressing fibronectin expression and $\beta 1$ integrin activation. Cell Physiol Biochem 27: 575-586, 2011.

21. Radford KJ, Thorne RF and Hersey P: CD63 associates with transmembrane 4 superfamily members, CD9 and CD81 and with beta 1 integrins in human melanoma. Biochem Biophys Res Commun 222: 13-18, 1996.

22. Si Z and Hersey P: Expression of the neuroglandular antigen and analogues in melanoma. CD9 expression appears inversely related to metastatic potential of melanoma. Int J Cancer 54: 37-43, 1993.

23. Sauer $\mathrm{T}$ and Suciu V: The role of preoperative axillary lymph node fine needle aspiration in locoregional staging of breast cancer. Ann Pathol 32: e24-e28, 2012.

24. Tran PV, Georgieff MK and Engeland WC: Sodium depletion increases sympathetic neurite outgrowth and expression of a novel TMEM35 gene-derived protein (TUF1) in the rat adrenal zona glomerulosa. Endocrinology 151: 4852-4860, 2010.

25. Livak KJ and Schmittgen TD: Analysis of relative gene expression data using real-time quantitative PCR and the 2(-Delta Delta C(T)) Method. Methods 25: 402-408, 2001.

26. Fromigué $\mathrm{O}$, Hamidouche $\mathrm{Z}$ and Marie PJ: Blockade of the RhoA-JNK-c-Jun-MMP2 cascade by atorvastatin reduces osteosarcoma cell invasion. J Biol Chem 283: 30549-30556, 2008.

27. Kuhn M, Szklarczyk D, Franceschini A, Campillos M, von Mering C, Jensen LJ, Beyer A and Bork P: STITCH 2: An interaction network database for small molecules and proteins. Nucleic Acids Res 38: D552-D556, 2010.

28. Hemler ME: Tetraspanin functions and associated microdomains. Nat Rev Mol Cell Biol 6: 801-811, 2005.

29. Whitelegge JP. Integral membrane proteins and bilayer proteomics. Anal Chem 85: 2558-2568, 2013.

30. Cobbold C, Monaco AP, Sivaprasadarao A and Ponnambalam S: Aberrant trafficking of transmembrane proteins in human disease. Trends Cell Biol 13: 639-647, 2003.

31. Pieck KI: More than $50 \%$ of drugs target membrane proteins. http://www.irbbarcelona.org/en/news/more-than-50-of-drugstarget-membrane-proteins (In Italian). Accessed December 30, 2014.

32. Ke AW, Shi GM, Zhou J, Huang XY, Shi YH, Ding ZB, Wang XY, Devbhandari RP and Fan J: CD151 amplifies signaling by integrin $\alpha 6 \beta 1$ to PI3K and induces the epithelial-mesenchymal transition in HCC cells. Gastroenterology 140: 1629-1641, e15, 2011.

33. Sadej R, Romanska H, Baldwin G, Gkirtzimanaki K, Novitskaya V, Filer AD, Krcova Z, Kusinska R, Ehrmann J, Buckley CD, et al: CD151 regulates tumorigenesis by modulating the communication between tumor cells and endothelium. Mol Cancer Res 7: 787-798, 2009 
34. Ke AW, Shi GM, Zhou J, Wu FZ, Ding ZB, Hu MY, Xu Y, Song ZJ, Wang ZJ, Wu JC, et al: Role of overexpression of CD151 and/or c-Met in predicting prognosis of hepatocellular carcinoma. Hepatology 49: 491-503, 2009.

35. Roland AV and Moenter SM. Regulation of gonadotropin-releasing hormone neurons by glucose. Trends Endocrinol Metab 22: 443-449, 2011.

36. Mantyh PW, Koltzenburg M, Mendell LM, Tive L and Shelton DL. Antagonism of nerve growth factor-TrkA signaling and the relief of pain. Anesthesiology 115: 189-204, 2011
37. Binder DK and Scharfman HE. Brain-derived neurotrophic factor. Growth Factors 22: 123-131, 2004.

38. Nilsson SF and Bill A. Vasoactive intestinal polypeptide (VIP): Effects in the eye and on regional blood flows. Acta Physiol Scand 121: 385-392, 1984

39. Rosenfeld CR, White RE, Roy T and Cox BE. Calcium-activated potassium channels and nitric oxide coregulate estrogen-induced vasodilation. Am J Physiol Heart Circ Physiol 279: H319-H312, 2000 . 